\title{
Polarization of the changing-look quasar $\mathrm{J} 1011+5442^{\star}$
}

\author{
D. Hutsemékers ${ }^{1, \star \star}$, B. Agís González ${ }^{1,2}$, D. Sluse ${ }^{1}$, C. Ramos Almeida ${ }^{3,4}$, and J.-A. Acosta Pulido 3,4 \\ 1 Institut d'Astrophysique et de Géophysique, Université de Liège, Allée du 6 Août 19c, B5c, 4000 Liège, Belgium \\ e-mail: hutsemekers@astro.ulg.ac.be \\ 2 Instituut voor Sterrenkunde, KU Leuven, Celestijnenlaan 200D, Bus 2401, 3001 Leuven, Belgium \\ 3 Instituto de Astrofisica de Canarias, Calle Via Lactea, s/n, 38205 La Laguna, Tenerife, Spain \\ 4 Departamento de Astrofisica, Universidad de La Laguna, 38205 La Laguna, Tenerife, Spain
}

Received 19 June 2017 / Accepted 13 July 2017

\begin{abstract}
If the disappearance of the broad emission lines observed in changing-look quasars were caused by the obscuration of the quasar core through moving dust clouds in the torus, high linear polarization typical of type 2 quasars would be expected. We measured the polarization of the changing-look quasar J1011+5442 in which the broad emission lines have disappeared between 2003 and 2015. We found a polarization degree compatible with null polarization. This measurement suggests that the observed change of look is not due to a change of obscuration hiding the continuum source and the broad line region, and that the quasar is seen close to the system axis. Our results thus support the idea that the vanishing of the broad emission lines in J1011+5442 is due to an intrinsic dimming of the ionizing continuum source that is most likely caused by a rapid decrease in the rate of accretion onto the supermassive black hole.
\end{abstract}

Key words. quasars: emission lines - quasars: general

\section{Introduction}

Type 1 quasars and lower luminosity active galactic nuclei (AGN) are characterized by broad emission lines (BELs) in their optical spectrum. These lines reveal high-velocity motions in the vicinity of the accretion disk that feeds the central supermassive black hole and emits the continuum spectrum. The high luminosity and strong outflows generated by quasar activity can be powerful enough to regulate star formation in the host galaxy and then play a significant role in their cosmological evolution (Silk \& Rees 1998; Borguet et al. 2013). The timescale of the activity cycle(s) in galaxies is thus a key parameter. Indirect measurements suggest that the quasar phenomenon is a relatively short stage in the evolution of galaxies, of the order of $10^{7-8} \mathrm{yr}$ (Martini \& Weinberg 2001). Schawinski et al. (2015) estimated AGN lifetimes of the order of $10^{5} \mathrm{yr}$, implying that black holes probably grow through short bursts. Quasars are then expected to "flicker" on and off.

Although transition objects are expected to be elusive, a handful of them may have been discovered recently. LaMassa et al. (2015) found the first quasar changing from type 1 (strong broad and narrow emission lines) to type 1.9 (strong narrow lines only and dim continuum). Soon after this, Runnoe et al. (2016) identified another quasar in which the BELs had disappeared. Thanks to systematic searches in the spectroscopic archive of the Sloan Digital Sky Survey (SDSS), Ruan et al. (2016) and MacLeod et al. (2016) uncovered about ten new changing-look quasars. The timescales of the changes are of the order of only a few years.

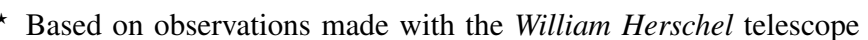
operated on the island of La Palma by the Isaac Newton Group of Telescopes in the Spanish Observatorio del Roque de los Muchachos of the Instituto de Astrofísica de Canarias.

$\star \star$ Senior Research Associate F.R.S.-FNRS
}

To explain these spectral changes, two main interpretations have been proposed. (I) Modifications in the source of ionizing radiation, likely a variation in accretion rate onto the supermassive black hole (Elitzur et al. 2014). An intrinsic dimming of the continuum source reduces the number of photons available to ionize the gas, resulting in a net decrease in emission line intensity. (II) Variable dust absorption along the observer's line of sight to the continuum source and the broad line region that is due to the motion of individual dust clouds in a clumpy torus (Goodrich 1989; Tran et al. 1992).

These two possible scenarios can be better distinguished in high-luminosity AGNs (quasars) because the inner BEL region is larger in this class, so that the lower limit on the crossing time of dust clouds consequently increases (LaMassa et al. 2015). The size of the broad line region is indeed proportional to $L^{\alpha}$ with $\alpha=0.5-0.7, L$ being the $5100 \AA$ optical luminosity (Kaspi et al. 2005; Bentz et al. 2006). It follows that the variable dust absorption scenario seems to be disfavoured in quasars because the extinction variation timescales that are due to dust clouds moving in front of the broad line region are factors 2-10 too long to explain the observed spectral changes (LaMassa et al. 2015; MacLeod et al. 2016). These results make changing-look quasars good candidates to be flickering AGN, and thus possible Rosetta stones for the study of quasar activity cycles.

In the present paper we use the low-/high-polarization dichotomy between type 1 and 2 quasars to further constrain these scenarios. In Sect. 2 we specify the polarization dichotomy between type 1 and 2 quasars in the redshift and luminosity ranges of interest. In Sect. 3 we report the polarization measurement of the changing-look quasar J1011+5442, in which broad emission lines have recently disappeared. We conclude in Sect. 4 that the null-polarization measured in $\mathrm{J} 1011+5442$ does not support the variable dust absorption scenario. 
Table 1. Polarization of type 2 and 1 quasars.

\begin{tabular}{|c|c|c|c|c|c|}
\hline Object & $z$ & Type & $p$ & $\sigma_{p}$ & Ref. \\
\hline $\mathrm{J} 081507.42+430427.2$ & 0.510 & 2 & 8.5 & 0.6 & Z05 \\
\hline J084234.94+362503.1 & 0.561 & $2^{*}$ & 16.5 & 0.2 & $\mathrm{Z} 05$ \\
\hline J092014.11+453157.2 & 0.402 & 2 & 4.7 & 0.9 & Z06 \\
\hline $\mathrm{J} 103951.49+643004.2$ & 0.402 & $2^{*}$ & 16.6 & 0.3 & Z05 \\
\hline $\mathrm{J} 110621.95+035747.1$ & 0.242 & 2 & 3.1 & 0.5 & Z06 \\
\hline J132323.33-015941.9 & 0.350 & 2 & 5.0 & 1.0 & $\mathrm{Z} 05$ \\
\hline $\mathrm{J} 140740.06+021748.3$ & 0.309 & 2 & 2.6 & 0.2 & $\mathrm{Z} 05$ \\
\hline $\mathrm{J} 141315.31-014221.0$ & 0.380 & 2 & 4.1 & 1.0 & $\mathrm{Z} 05$ \\
\hline $\mathrm{J} 150608.09-020744.2$ & 0.439 & 2 & 9.7 & 1.1 & Z05 \\
\hline $\mathrm{J} 151711.47+033100.2$ & 0.613 & 2 & 10.5 & 0.8 & Z05 \\
\hline $\mathrm{J} 154340.02+493512.6$ & 0.512 & $2^{*}$ & 7.7 & 0.3 & $\mathrm{Z} 05$ \\
\hline J164131.73+385840.9 & 0.596 & $2^{*}$ & 4.5 & 0.3 & $\mathrm{Z} 05$ \\
\hline $\mathrm{J} 171559.79+280716.8$ & 0.524 & $2^{*}$ & 3.5 & 0.3 & $\mathrm{Z} 05$ \\
\hline J014017.06-005003.0 & 0.334 & $\overline{1^{\dagger}}$ & 0.63 & 0.31 & $\begin{array}{l}\text { S84 } \\
\end{array}$ \\
\hline VJ015130.8+091725 & 0.299 & 1 & 1.21 & 0.54 & M84 \\
\hline J095048.38+392650.4 & 0.205 & $1^{\dagger}$ & 0.19 & 0.15 & B90 \\
\hline $\mathrm{J} 095652.39+411522.2$ & 0.234 & 1 & 0.25 & 0.22 & B90 \\
\hline $\mathrm{J} 105151.44-005117.6$ & 0.359 & $1^{\dagger}$ & 0.57 & 0.28 & B90 \\
\hline $\mathrm{J} 112439.18+420145.0$ & 0.225 & $1^{\dagger}$ & 0.44 & 0.19 & B90 \\
\hline $\mathrm{J} 113230.09-024620.9$ & 0.332 & 1 & 0.53 & 0.16 & S05 \\
\hline $\mathrm{J} 113432.29-005548.1$ & 0.268 & $1^{\dagger}$ & 0.42 & 0.18 & S05 \\
\hline $\mathrm{J} 121920.93+063838.5$ & 0.331 & 1 & 0.60 & 0.13 & S05 \\
\hline $\mathrm{J} 121946.53+145259.3$ & 0.401 & $1^{\dagger}$ & 0.52 & 0.26 & S05 \\
\hline $\mathrm{J} 124654.03+131310.7$ & 0.512 & $1^{\dagger}$ & 0.14 & 0.14 & S05 \\
\hline $\mathrm{J} 124730.96-014227.7$ & 0.346 & $1^{\dagger}$ & 1.12 & 0.36 & S05 \\
\hline $\mathrm{J} 130112.91+590206.6$ & 0.477 & 1 & 0.06 & 0.17 & B90 \\
\hline $\mathrm{J} 134251.60-005345.3$ & 0.326 & $1^{\dagger}$ & 0.21 & 0.14 & S05 \\
\hline $\mathrm{J} 135632.79+210352.3$ & 0.300 & $1^{\dagger}$ & 1.42 & 0.31 & B90 \\
\hline $\mathrm{J} 140031.96+040457.6$ & 0.428 & $1^{\dagger}$ & 0.47 & 0.27 & S84 \\
\hline $\mathrm{J} 142943.07+474726.2$ & 0.220 & $1^{\dagger}$ & 0.25 & 0.29 & B90 \\
\hline $\mathrm{J} 144645.93+403505.7$ & 0.267 & 1 & 0.37 & 0.15 & B90 \\
\hline $\mathrm{J} 154530.23+484609.0$ & 0.401 & 1 & 0.08 & 0.20 & B90 \\
\hline $\mathrm{J} 161410.62+263250.4$ & 0.395 & 1 & 1.24 & 0.56 & S84 \\
\hline VJ214423.0+041627 & 0.463 & 1 & 0.84 & 0.25 & S05 \\
\hline VJ215819.1+020849 & 0.560 & 1 & 0.51 & 0.22 & S05 \\
\hline VJ222049.7-315654 & 0.506 & 1 & 0.55 & 0.28 & S05 \\
\hline VJ232210.8-344757 & 0.420 & 1 & 0.32 & 0.22 & S05 \\
\hline VJ234844.8-361827 & 0.541 & 1 & 0.64 & 0.25 & S05 \\
\hline VJ235729.3+002940 & 0.410 & 1 & 0.67 & 0.30 & S05 \\
\hline
\end{tabular}

Notes. Object name and coordinates are either from SDSS or from Véron-Cetty \& Véron (2001), the latter with prefix V. The linear polarization degree $p$ and its error $\sigma_{p}$ are given in $\%$. ${ }^{(*)}$ Type 2 quasars for which broad emission lines have been detected in the polarized light. (†) Type 1 quasars with $L_{[\mathrm{O} \text { III }]} \gtrsim 3 \times 10^{8} L_{\odot}$.

References. (Z05) Zakamska et al. (2005); (Z06) Zakamska et al. (2006); (S84) Stockman et al. (1984); (M84) Moore \& Stockman (1984); (B90) Berriman et al. (1990); (S05) Sluse et al. (2005).

\section{Polarization of type 1 and 2 quasars}

Quasar light is known to be linearly polarized at optical wavelengths, with significant differences between type 1 and 2 objects, the latter showing the highest polarizations.

Zakamska et al. (2003) have built a large sample of type 2 quasars from the spectroscopic data of the Sloan Digital Sky Survey. These objects are optically faint, with no broad permitted lines, and show narrow emission lines with high equivalent widths. Since the broad line region and the source of continuum are obscured, the luminosity of the [O III] lines, assumed to come from an extended and therefore unobscured region, is used as a proxy of the intrinsic luminosity. Since luminous $\left(M_{\mathrm{B}}<-23\right)$ type 1 quasars have, on average, [O III] $\lambda 5007$ line luminosities higher than $3 \times 10^{8} L_{\odot}$, this value was used as a cutoff to identify intrinsically luminous type 2 objects (Zakamska et al. 2003). Subsequent spectropolarimetry (Zakamska et al. 2005, 2006) has revealed highly polarized quasars with broad lines in their polarized spectrum, which clearly proved that these type 2 quasars indeed harbour a luminous quasar in their highly obscured core. In Table 1 we have collected the linear polarization degree ( $p$ and its error $\sigma_{p}$ in $\%$ ) of the radio-quiet type 2 quasars studied in Zakamska et al. (2005, 2006). Only the restframe UV-blue (2820-3710 ̊) polarization measurements are considered here. All these measurements have $p / \sigma_{p}>4$. The sample contains 13 type 2 quasars with redshifts $z$ between 0.2 and 0.7 .

To build a comparison sample of type 1 quasar polarizations, we used the data set compiled by Hutsemékers et al. (2005). We restricted the sample to good-quality polarization data, that is, we only considered objects with either $p \geq 0.6 \%$ and $p / \sigma_{p} \geq 2$, or $p<0.6 \%$ and $\sigma_{p}<0.3 \%$. To minimize contamination by interstellar polarization, we also selected objects at high Galactic latitudes $\left|b_{\text {gal }}\right| \geq 30^{\circ}$. Radio-loud and red quasars were discarded, as were broad absorption line quasars, the polarization of which differs from that of normal radio-quiet objects (Moore \& Stockman 1984; Hutsemékers et al. 1998; Smith et al. 2002). The final sample, matching the redshift range of type 2 quasars $0.2<z<0.7$, contains 26 radio-quiet type 1 quasars (Table 1). All these objects are type 1 quasars with $M_{\mathrm{B}}<$ -23 (Véron-Cetty \& Véron 2001). Fourteen of them have measured [O III] $\lambda 5007$ luminosities that are between $1 \times 10^{8}$ and $8 \times 10^{9} L_{\odot}$ (Shen et al. 2011), 12 of them satisfying $L_{[\mathrm{O} \text { III }]} \gtrsim$ $3 \times 10^{8} L_{\odot}$ within the uncertainties.

The distributions of type 1 and 2 quasar polarizations are illustrated in Fig. 1. A clear dichotomy is seen: all type 1 quasars have low polarizations, in agreement with previous studies (e.g. Berriman et al. 1990), while all type 2 quasars have high polarizations $p>2 \%$. It is important to note that the polarization measurements of type 1 quasars reported in Table 1 were obtained in the $V$ filter (mean $\lambda \simeq 555 \mathrm{~nm}$; Sluse et al. 2005) or in "white light" (mean $\lambda \simeq 590 \mathrm{~nm}$; Stockman et al. 1984; Moore \& Stockman 1984; Berriman et al. 1990), while the type 2 quasar polarizations obtained by Zakamska et al. (2005, 2006) in the 2820-3710 $\AA$ rest-frame wavelength range correspond to $g$-band observations (mean $\lambda \simeq 475 \mathrm{~nm}$ for an average redshift of 0.45 ). Since the polarization of type 1 quasars may slightly depend on wavelength as $p(\lambda) \propto \lambda^{\alpha}$ with $\alpha=$ $-0.7 \pm 0.1$ (Stockman et al. 1984), we expect the $g$-band polarization of type 1 quasars to be at most $20 \%$ higher than the values reported in Table 1 . This is negligible with respect to the observed type 1/type 2 difference. A similar dichotomy is observed for lower luminosity Seyfert 1 and 2 AGNs (Marin 2014), although it is not as sharp as for quasars. For lower luminosity objects with lower redshift, this might be due to higher contamination by the host galaxy and the fact that polarization is measured at longer rest-frame wavelengths ${ }^{1}$.

Following the models initially developped for Seyferts (e.g. Smith et al. 2004; Batcheldor et al. 2011; Marin 2014), the

\footnotetext{
1 In addition, some Seyfert 1 AGNs show intermediate polarizations (polar-scattered Seyfert 1). Their high-luminosity counterparts might be broad absorption line quasars (Smith et al. 2004), which have been discarded from our comparison sample.
} 


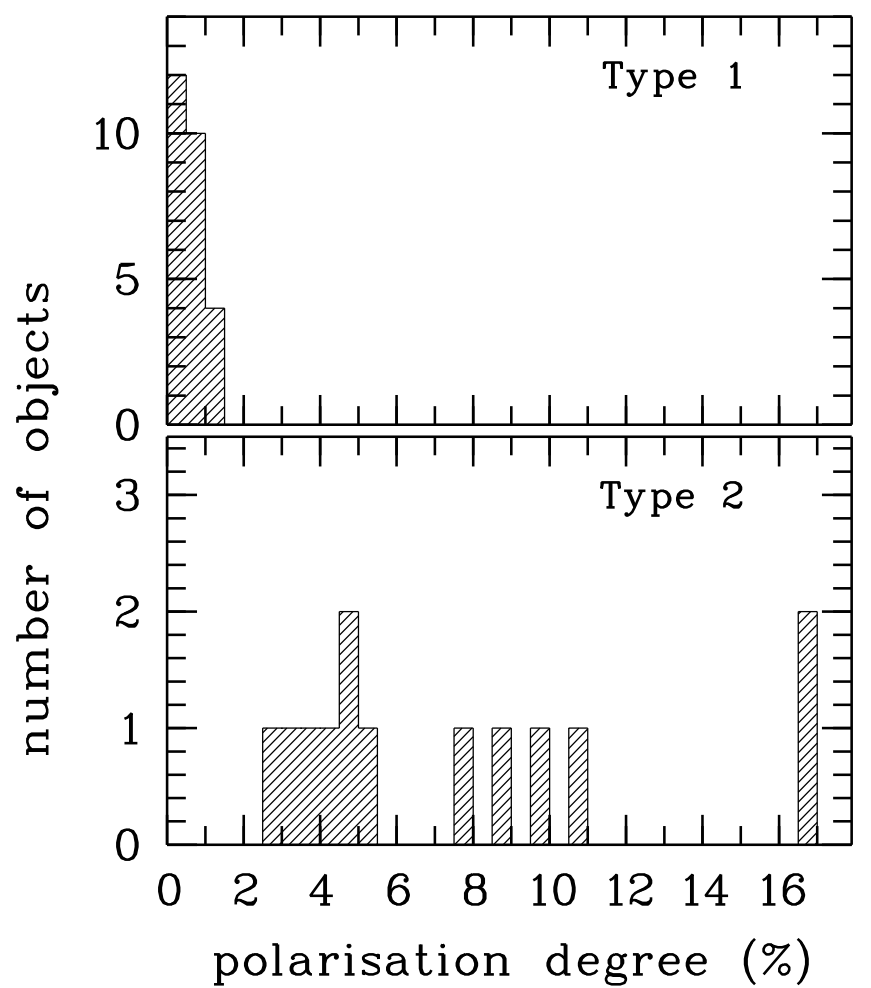

Fig. 1. Distribution of the rest-frame UV-blue linear polarization in type 1 and 2 radio-quiet quasars with redshifts $0.2<z<0.7$. All type 1 quasars in that redshift range have $p<2 \%$, while all type 2 quasars have $p>2 \%$.

polarization of radio-quiet quasars can be interpreted by scattering off two regions: an equatorial ring located inside the dusty torus at the origin of "parallel" polarization, and a more extended polar-scattering region at the origin of "perpendicular" polarization (e.g. Zakamska et al. 2005; Borguet et al. 2008). In type 1 quasars, seen at low inclinations (the angle between the system axis and the line of sight), the continuum source and both scattering regions are seen by the observer, resulting in a low polarization. In type 2 quasars, seen at high inclinations, the quasar core is hidden by the torus and only highly polarized polar-scattered light is seen. A change in polarization properties is thus expected from type 1 to type 2 depending on inclination, as is observed in Seyfert galaxies (Smith et al. 2004; Marin 2014). A change in polarization is also expected if the torus properties are varying, as shown by Marin et al. (2016). If the disappearance of broad emission lines in changing-look quasars is caused by torus dust clouds hiding the quasar core (i.e. the continuum source, the BEL region, and the equatorial scattering region), the high polarization typical of type 2 quasars is expected in quasars in which broad emission lines have disappeared.

\section{Polarization of $\mathrm{J} 1011+5442$}

SDSS J101152.98+544206.4 (hereafter J1011+5442) is a changing-look quasar discovered by Runnoe et al. (2016). SDSS photometric observations of J1011+5442 were obtained on February 13, 2002, and spectroscopic observations on January 13, 2003. These data led to the classification of $\mathrm{J} 1011+5442$ as a broad-line radio-quiet type 1 quasar of redshift $z=0.246$ and absolute magnitude $M_{i}=-22.87$ (Schneider et al. 2005; 2010; Shen et al. 2011). J1011+5442 was re-observed spectroscopically on February 20, 2015, as part of the Time-Domain
Spectroscopic Survey (Morganson et al. 2015), showing a complete disappearance of the strong broad $\mathrm{H} \beta$ line (Runnoe et al. 2016). This spectroscopic change from type 1 to type 1.9 (faint broad $\mathrm{H} \alpha$ is still detected) is accompanied by a systematic decline of the light curve from 2002 to 2015 (Runnoe et al. 2016). In particular, MacLeod et al. (2016) reported a brightness decrease from SDSS $g=18.37$ in February 2002 to Pan-STARRS1 $g=19.88$ in October 2011. A similar brightness change is found when comparing the Gaia $G$ magnitude computed from the SDSS magnitudes in February 2002 using the colour transformation of Jordi et al. (2010), that is, $G=18.03$, to the magnitude reported in the Gaia Data Release 1 (Gaia Collaboration 2016), $G=19.91$, which is the average of 40 observations between July 2014 and September 2015.

We obtained new linear polarization data of J1011+5442 on February 19, 2017, using the Intermediate dispersion Spectrograph and Imaging System (ISIS) mounted at the Cassegrain focus of the $4.2 \mathrm{~m}$ William Herschel Telescope (WHT) at the Roque de los Muchachos Observatory. Observations were made through the blue arm and the Sloan Gunn $g$ filter (ING filter $\# 218 ; \lambda_{c}=4844 \AA, F W H M=1280 \AA$ ), with ISIS in its imaging polarimetry mode. Four exposures of 15 minutes each with the half-wave plate at $8^{\circ}, 30.5^{\circ}, 53^{\circ}$, and $75.5^{\circ}$ were secured. This sequence was repeated, but the last exposure was aborted due to increasing humidity. After bias subtraction and flat-fielding the frames, the Stokes parameters were measured using the procedures described in Sluse et al. (2005). Polarized and unpolarized standard stars (HD 283812, HD 251204, and G191B2B from Turnshek et al. 1990) were observed to correct for the chromatic dependence of the half-wave plate zero-angle and to estimate the instrumental polarization, respectively. The linear polarization degree of $\mathrm{J} 1011+5442$ in the rest-frame $3200-4400 \AA$ wavelength range is found to be $p=0.15 \%$ with an error $\sigma_{p}=0.22 \%$. Since $\sigma_{p}>p$, the debiased polarization degree is equal to zero and the polarization position angle cannot be estimated with reasonable accuracy. The instrumental polarization is measured equal to $p=0.07 \pm 0.04 \%$. Based on the foreground Galactic extinction from Schlafly \& Finkbeiner (2011) given in the NASA/IPAC Extragalactic Database (NED), the interstellar polarization towards $\mathrm{J} 1011+5442$ is expected to be $p \leq 9 \% \times E(B-V) \simeq 0.1 \%$. The polarization of $\mathrm{J} 1011+5442$ is therefore compatible with a null intrinsic linear polarization.

To check the magnitude of J1011+5442 in February 2017, we used a faint star in the ISIS field of view that is located in one open slot of the mask: SDSS J101153.55+544111.1, which has a magnitude $g($ SDSS $)=22.1 \pm 0.1$. By stacking all frames obtained in the polarimetric mode, we measured $F_{\text {quasar }} / F_{\text {star }}=$ $10.5 \pm 1.0$ in the WHT $g$ filter. This translates into $g \simeq 19.6 \pm 0.2$ for $\mathrm{J} 1011+5442$. Although this is a rough estimate, it indicates that J1011+5442 was still in its faint state in February 2017.

\section{Discussion and conclusion}

Although the quasar $\mathrm{J} 1011+5442$ is in a type 2 dim state, its polarization appears to be very low, typical of unobscured type 1 quasars. This suggests that the observed change of look is not due to a change of obscuration in the torus hiding the continuum source, the broad line region, and the equatorial scattering region. The null polarization also suggests that the quasar is seen at low inclination so that the scattering regions appear essentially symmetric, that is, far from lines of sight crossing the dusty torus. Our results thus support the idea that the vanishing of the broad emission lines is due to an intrinsic dimming of 
the ionizing continuum source most likely caused by a rapid decrease of the rate of accretion onto the supermassive black hole, in agreement with the conclusions reached independently by LaMassa et al. (2015), Runnoe et al. (2016), and MacLeod et al. (2016). In this scenario, the brightness decrease of the continuum source would be accompanied by a similar decrease of the scattered flux, with no significant change of the polarization degree. A time delay is nevertheless expected between the variations of the direct and scattered light, depending on the sizes of the equatorial and polar scattering regions. The size of the equatorial scattering region being comparable to that of the broad line region, the time delay is about one month in $\mathrm{J} 1011+5442$ (Runnoe et al. 2016), which is short with respect to the timescale of the change of look (years). The size of the polar scattering region, on the other hand, is much larger. While the time delay between the direct and polar-scattered light remains small at low inclination, it could then be larger at intermediate inclinations, possibly generating complex polarization variations. This is the basis of polarization reverberation mapping (e.g. Gaskell et al. 2012).

Polarimetry of changing-look quasars thus appears useful not only to distinguish the mechanisms at the origin of quasar type changes, but also to constrain the properties of the scattering regions. This implicitly assumes that changing-look quasars, and $\mathrm{J} 1011+5442$ in particular, do possess the scattering regions found in other radio-quiet quasars. A monitoring of the linear polarization of a significant sample of changing-look quasars is thus needed.

Acknowledgements. D.H. and B.A.G. thank Marie Hrudkova for her help during the observations. C.R.A. acknowledges the Ramón y Cajal Program of the Spanish Ministry of Economy and Competitiveness through project RYC-2014-15779 and the Spanish Plan Nacional de Astronomía y Astrofisíca under grant AYA2016-76682-C3-2-P. This research has made use of the NASA/IPAC Extragalactic Database (NED) which is operated by the Jet Propulsion Laboratory, California Institute of Technology, under contract with the National Aeronautics and Space Administration. This work has made use of data from the European Space Agency (ESA) mission Gaia (https://www . cosmos.esa.int/gaia), processed by the Gaia Data Processing and Analysis Consortium (DPAC, https://www.cosmos.esa.int/web/gaia/dpac/ consortium). Funding for the DPAC has been provided by national institutions, in particular the institutions participating in the Gaia Multilateral Agreement.

\section{References}

Batcheldor, D., Robinson, A., Axon, D. J., et al. 2011, ApJ, 738, 90 Bentz, M. C., Peterson, B. M., Pogge, R. W., Vestergaard, M., \& Onken, C. A. 2006, ApJ, 644, 133

Berriman, G., Schmidt, G. D., West, S. C., \& Stockman, H. S. 1990, ApJS, 74, 869

Borguet, B., Hutsemékers, D., Letawe, G., Letawe, Y., \& Magain, P. 2008, A\&A, 478,321

Borguet, B. C. J., Arav, N., Edmonds, D., Chamberlain, C., \& Benn, C. 2013, ApJ, 762, 49

Elitzur, M., Ho, L. C., \& Trump, J. R. 2014, MNRAS, 438, 3340

Gaia Collaboration (Brown, A. G. A., et al.) 2016, A\&A, 595, A2

Gaskell, C. M., Goosmann, R. W., Merkulova, N. I., Shakhovskoy, N. M., \& Shoji, M. 2012, ApJ, 749, 148

Goodrich, R. W. 1989, ApJ, 340, 190

Hutsemékers, D., Lamy, H., \& Remy, M. 1998, A\&A, 340, 371

Hutsemékers, D., Cabanac, R., Lamy, H., \& Sluse, D. 2005, A\&A, 441, 915

Jordi, C., Gebran, M., Carrasco, J. M., et al. 2010, A\&A, 523, A48

Kaspi, S., Maoz, D., Netzer, H., et al. 2005, ApJ, 629, 61

LaMassa, S. M., Cales, S., Moran, E. C., et al. 2015, ApJ, 800, 144

MacLeod, C. L., Ross, N. P., Lawrence, A., et al. 2016, MNRAS, 457, 389

Marin, F. 2014, MNRAS, 441, 551

Marin, F., Goosmann, R. W., \& Petrucci, P.-O. 2016, A\&A, 591, A23

Martini, P., \& Weinberg, D. H. 2001, ApJ, 547, 12

Moore, R. L., \& Stockman, H. S. 1984, ApJ, 279, 465

Morganson, E., Green, P. J., Anderson, S. F., et al. 2015, ApJ, 806, 244

Ruan, J. J., Anderson, S. F., Cales, S. L., et al. 2016, ApJ, 826, 188

Runnoe, J. C., Cales, S., Ruan, J. J., et al. 2016, MNRAS, 455, 1691

Schawinski, K., Koss, M., Berney, S., \& Sartori, L. F. 2015, MNRAS, 451, 2517

Schlafly, E. F., \& Finkbeiner, D. P. 2011, ApJ, 737, 103

Schneider, D. P., Hall, P. B., Richards, G. T., et al. 2005, AJ, 130, 367

Schneider, D. P., Richards, G. T., Hall, P. B., et al. 2010, AJ, 139, 2360

Shen, Y., Richards, G. T., Strauss, M. A., et al. 2011, ApJS, 194, 45

Silk, J., \& Rees, M. J. 1998, A\&A, 331, L1

Sluse, D., Hutsemékers, D., Lamy, H., Cabanac, R., \& Quintana, H. 2005, A\&A, 433, 757

Smith, J. E., Robinson, A., Alexander, D. M., et al. 2004, MNRAS, 350, 140

Smith, P. S., Schmidt, G. D., Hines, D. C., Cutri, R. M., \& Nelson, B. O. 2002, ApJ, 569, 23

Stockman, H. S., Moore, R. L., \& Angel, J. R. P. 1984, ApJ, 279, 485

Tran, H. D., Osterbrock, D. E., \& Martel, A. 1992, AJ, 104, 2072

Turnshek, D. A., Bohlin, R. C., Williamson, II, R. L., et al. 1990, AJ, 99, 1243

Véron-Cetty, M.-P., \& Véron, P. 2001, A\&A, 374, 92

Zakamska, N. L., Schmidt, G. D., Smith, P. S., et al. 2005, AJ, 129, 1212

Zakamska, N. L., Strauss, M. A., Krolik, J. H., et al. 2003, AJ, 126, 2125

Zakamska, N. L., Strauss, M. A., Krolik, J. H., et al. 2006, AJ, 132, 1496 